\title{
Depression and Brain-Derived Neurotrophic Factor Levels in Alzheimer's Disease
}

\author{
James R. Hall ${ }^{1,2}$, Sid E. O’Bryant ${ }^{3,4}$, Leigh Johnson ${ }^{5,6}$, Robert C. Barber ${ }^{7}$ \\ ${ }^{1}$ Institute of Aging and Alzheimer's Disease Research, University of North Texas Health Sciences Center, Fort Worth, TX, USA; \\ ${ }^{2}$ Department of Psychiatry, University of North Texas Health Sciences Center, Fort Worth, TX, USA; ${ }^{3}$ F. Marie Hall Institute for \\ Rural \& Community Health, Texas Tech Health Sciences Center, Lubbock, TX, USA; ${ }^{4}$ Department of Neurology, Texas Tech Uni- \\ versity Health Sciences Center, Lubbock, TX, USA; ${ }^{5}$ Laura W. Bush Institute for Women's Health, Texas Tech University Health \\ Sciences Center, Amarillo, TX, USA; ${ }^{6}$ Department of Family and Community Medicine, Texas Tech University Health Sciences \\ Center, Lubbock, TX, USA; ${ }^{7}$ Department of Pharmacology and Neuroscience, University of North Texas Health Sciences Center, \\ Fort Worth, TX, USA. \\ Email: james.hall@unthsc.edu
}

Received January $12^{\text {th }}, 2011$; revised January $24^{\text {th }}, 2011$; accepted March $6^{\text {th }}, 2011$.

\begin{abstract}
Background: Depression is often viewed as a risk factor for the development of Alzheimer's disease (AD), however little is known regarding the underlying biological mechanisms linking these two diseases. Brain-derived neurotrophic factor $(B D N F)$ has been linked to both cognitive impairment and depression in past research; however few studies have examined this relation in a sample of Alzheimer's patients. The present study sought to address this gap in the literature by examining the relation between serum BDNF levels and depression assessed by the Geriatric Depression Scale (GDS) in a group of patients diagnosed with probable Alzheimer's disease. Methods: Participants included 169 individuals diagnosed with Probable AD enrolled in the TARC Longitudinal Research Cohort with available BDNF levels and GDS scores. The participants were divided into Depressed $(N=20)$ and Not Depressed $(N=149)$ based on GDS scores. Results: BDNF levels significantly predicted level (High vs. Low) of depression $(\beta=0.066, S E=0.031, p=0.034)$. BDNF levels for the Depressed group were significantly higher than those observed in the Not Depressed group $(p>0.036)$. Conclusions: These findings suggest that an upregulation of BDNF possibly exists among depressed AD patients as a response to the chronic inflammatory processes that occur in depression. This upregulation of BDNF appears to persist at least into early stages of Alzheimer's.
\end{abstract}

Keywords: Alzheimer's Disease, Depression, BDNF, Depression Severity

\section{Introduction}

Depression has been identified as a risk factor for the development of Alzheimer's disease [1], as a factor in the conversion of $\mathrm{MCI}$ to $\mathrm{AD}$ [2], and as a disorder affecting a significant number of individuals with AD [3]. The underlying biological mechanisms linking depression and $\mathrm{AD}$ are unclear. Among the mechanisms proposed has been the dysregulation of neurotrophin signaling which has been found in both depression [4] and AD [5]. Neurotrophins are small proteins that play significant roles in maintaining neuronal survival and axonal guidance. Brain-derived neurotrophic factor (BDNF) is one neurotrophin whose level has been linked to impaired cognition, depression and $\mathrm{AD}[6]$.

Lower serum BDNF levels have been reported in patients with major depression when compared to normals
$[7,8]$ and decreased levels of BDNF have been reported in Alzheimer's patients when compared with cognitively normal subjects $[9,10]$ although a recent study found no differences [11]. Few studies have investigated the relationship between BDNF and depression in Alzheimer's patients. In one such study, a negative correlation between BDNF serum concentrations and level of depression as measured by the Geriatric Depression Scale has been reported [12]. In contrast, a study of elderly Korean $\mathrm{AD}$ patients found no differences in serum BDNF levels between subjects scoring high on the GDS $(\geqslant 20)$ and those scoring low on the GDS [13]. The current study investigated the relationship between serum BDNF concentrations and level of depression as measured by the GDS in a sample of Alzheimer's patients to help clarify this relationship. 


\section{Methods}

\subsection{Participants}

Participants included 169 individuals diagnosed with Probable AD enrolled in the TARC Longitudinal Research Cohort with available BDNF levels and GDS scores. Individuals with a history of a Major Depressive Disorder, a concurrent Major Depressive Disorder or a depression significant enough to impact neuropsychological test performance were excluded from the TARC cohort. The methodology of the TARC project has been described in detail elsewhere [14]. Briefly, each participant completes an annual examination consisting of a medical examination, interview, blood draw, and neuropsychological testing at one of the five TARC sites. These data are reviewed by a consensus committee at each site and a diagnosis is assigned according to NINCDS-ADRDA criteria [15]. Only those meeting criteria for probable AD are included in the AD sample. The characteristics of the participants are shown in Table $\mathbf{1 .}$ The TARC cohort also consists of a sample of elderly individuals that were judged to be within normal limits by consensus on cognitive measures and the GDS. This sample of 198 controls without depression was included as a comparison sample for post hoc analyses. The TARC project received Institutional Review Board approval and all participants and/or caregivers signed written informed consent documents.

\subsection{Measures}

The TARC neuropsychology core battery consists of common instruments administered as part of the established Alzheimer's disease clinical/research platforms at each participating institution and includes digit span (WAIS-R, WAIS-III, WMS-R), Trail Making Test, WMS Logical Memory and Visual Reproduction (WMS-R and WMS-III), Boston Naming Test (30- and 60-item versions), verbal fluency (FAS), Clock Drawing Test, the American National Adult Reading Test (AMNART), the Geriatric Depression Scale (GDS-30), Mini-Mental State Examination (MMSE), and ratings on the Clinical Dementia Rating scale (CDR).

\subsection{Assays}

Non-fasting samples were collected in serum-separating tubes during clinical evaluations, allowed to clot at room temperature for 30 minutes, centrifuged, aliquoted, and stored at $-80^{\circ} \mathrm{C}$ in plastic vials. Samples were sent frozen to Rules Based Medicine (www.rulesbasedmedicine.com, Austin, TX) where they were thawed for assay without additional freeze-thaw cycles. Rules Based Medicine conducted multiplexed immunoassays via their human Multi-Analyte Profile (human MAP). Multiple proteins, including BDNF, were quantified though multiplex fluorescent immunoassay utilizing colored microspheres with protein-specific antibodies. For BDNF, the least detectable dose (LDD) was $0.029 \mathrm{ng} / \mathrm{mL}$, inter-run coefficient of variation was $<7 \%$, dynamic range was $0.0028-14$ $\mathrm{ng} / \mathrm{mL}$, overall spiked standard recovery was $95 \%$, and cross-reactivity with other human MAP analytes was $<5 \%$. Assays of BDNF levels from TARC data conducted by this company utilizing this platform have been published elsewhere [11].

\subsection{Analyses}

Statistical analyses were conducted using SPSS version 17 (Chicago, Ill). The participants were divided into two groups based on scores on the GDS; Not Depressed Group (GDS Score $\leqslant 9$ ) and Depressed Group (GDS Score $\geqslant 10$ ). Analyses comparing the demographic characteristics of the sample were conducted using t-tests for continuous and chi-squared test for discrete variables. In order to determine if serum BDNF levels were significantly related to level of depression a binary logistic regression was applied with BDNF as the predictor variable and level of depression (High vs. Low) as the outcome variable. This logistic regression model included age, gender, education and CDR score as covariates. To further investigate differences between the groups, Univariant Analysis of Variance was conducted with BDNF as the dependent variable. CDR Global score was used a co-variant to control for any affect of stage of disease in analyzing level of depression and BDNF levels. A t-test for independent samples was used in the post hoc analysis to compare non-depressed $\mathrm{AD}$ with a sample of non-depressed controls. Statistical significance was set as a p-value $<0.05$.

\section{Results}

Demographic characteristics of the participants are shown in Table 1. There were no significant differences between the Not Depressed group and the Depressed group on age or education. There were more females than males in each group, although the gender distribution was not significantly different between groups. The sample was primarily Caucasian (96\%). The two groups did not differ on MMSE. As would be expected from the selection procedure of dividing participants into Depressed and Not Depressed groups based on a cutoff score, the two groups differed significantly on GDS score $(\mathrm{p}>0.001)$. There were no significant differences in BDNF levels between depressed and non-depressed patients for total GDS score. Results of the logistic regression found that BDNF significantly predicted severity (High vs. Low) of depression $(\beta=0.066, \mathrm{SE}=0.031, \mathrm{p}=$ 0.034 ) in a model including age, gender, education and 
Table 1. Characteristics of the participants.

\begin{tabular}{|c|c|c|c|}
\hline & Not Depressed $(\mathrm{N}=149)$ & Depressed $(\mathrm{N}=20)$ & $\mathrm{p}$ \\
\hline \multicolumn{4}{|l|}{ SEX } \\
\hline Females & $98(66 \%)$ & $13(65 \%)$ & 0.882 \\
\hline Males & $51(34 \%)$ & $7(35 \%)$ & \\
\hline \multicolumn{4}{|l|}{ AGE } \\
\hline Mean (SD) & $77.72(7.80)$ & $76.05(9.57)$ & 0.717 \\
\hline Range & $57-92$ & $58-91$ & \\
\hline \multicolumn{4}{|c|}{ EDUCATION } \\
\hline Mean (SD) & $14.3(3.488)$ & $12.75(4.102)$ & 0.427 \\
\hline Range & $0-25$ years & 5-22 years & \\
\hline \multicolumn{4}{|l|}{ MMSE } \\
\hline Mean (SD) & $20.07(5.323)$ & $21.05(5.083)$ & 0.718 \\
\hline Range & $4-30$ & 4-30 & \\
\hline \multicolumn{4}{|l|}{ GDS 30} \\
\hline Mean (SD) & $3.69(2.676)$ & $15.55(3.649)$ & $<0.001^{*}$ \\
\hline Range & $0-9$ & $11-22$ & \\
\hline \multicolumn{4}{|l|}{ BDNF } \\
\hline Mean (SD) & $30.91(9.172)$ & $36.17(7.272)$ & $0.036^{* *}$ \\
\hline Range & $12-44$ & $23-44$ & \\
\hline
\end{tabular}

Table 2. Odds ratios for factors associated with depression among Alzheimer's disease patients as determined by logistic regression. $\mathrm{N}=169$.

\begin{tabular}{lcccc}
\hline \multicolumn{1}{c}{ Risk Factor } & $\begin{array}{l}\text { Odds } \\
\text { Ratio }\end{array}$ & \multicolumn{2}{c}{$\begin{array}{c}\text { 95\% CI for } \\
\text { Odds Ratio }\end{array}$} & P-value \\
\hline Age & 0.967 & 0.909 & 1.030 & 0.299 \\
Gender & 1.031 & 0.362 & 2.939 & 0.955 \\
Education & 0.886 & 0.779 & 1.007 & 0.064 \\
CDR Score & 0.597 & 0.263 & 1.359 & 0.219 \\
BDNF & 1.068 & 1.005 & 1.135 & $0.034^{*}$ \\
\hline$* \mathrm{p}<0.05$ & & & &
\end{tabular}

CDR Global Impairment (Table 2). The BDNF levels for the Depressed group were significantly higher than those found for the Not Depressed group $(F=4.485, p=0.036)$ when the affect of stage of disease was co-varied.

\section{Discussion}

Although there has been little research on BDNF and depression in Alzheimer's disease, what research that has been conducted, along with the more extensive research on BDNF and depression and BDNF and AD, suggests that a decline in serum concentration in depressed AD patients would be expected. The current study found a significantly higher serum BDNF level for AD patients scoring in the depressed range on the GDS when com- pared with not depressed AD patients. This is contradictory to the findings of a previous study that used the GDS and found a significant negative correlation between GDS scores and BDNF levels [12]. The results from the previous study, however, were limited by the small number of AD patients assessed $(\mathrm{N}=11)$. Another study found no difference in BDNF levels when comparing severely depressed patients with those not severely depressed [13]. That study compared patients with age associated cognitive decline, MCI and AD and used 20 as the cutoff for severe depression. The overall level of depression in the sample was very high with the means of each group being near or above 20. Although the ranges for the GDS scores were not given, it is likely 
that many of the "not severely depressed" would have met the criteria for depression used in our study. Additionally, the sample included MCI patients and control, whereas only AD patients were used in the current study.

Although the findings are contradictory to some previous findings on the relationship of BDNF to GDS scores, they are consistent with others showing an increase in BDNF early in Alzheimer's [16] and studies reporting an increase in BDNF in both MCI and Alzheimer's [17]. Our sample consisted of AD patients in the mild stage of dementia and this increased level of BDNF may be a compensatory upregulation to deal with the accumulation of Alzheimer's pathology. To further investigate the role of depression a post hoc analysis compared the non-depressed normals (BDNF Mean = 30.83 , SD $=8.947)$ in the TARC sample with the non-depressed $\mathrm{AD}$ group. There was no difference on BDNF levels $(t=0.179, p=0.869)$. This suggests that depression is a contributing factor to the increased BDNF level. The underlying mechanism responsible for the higher BDNF level in the depressed group may be a reflection of chronic inflammatory processes that are related to the pathophysiology of depression [18] and persist in early Alzheimer's. Inflammation has long been implicated as a pathogenic mechanism in AD. O'Bryant and colleagues [19] recently developed a highly predictive algorithm for Alzheimer's disease based on concentrations of serum proteins that was strongly influenced by markers of inflammation. The authors concluded that “... a disproportionate number of inflammatory and vascular markers were weighted most heavily in analyses. Additionally, these markers consistently distinguished cases from controls...suggesting the existence of an inflammatory-related endophenotype of AD." The present findings likewise suggest an inflammatory- related endophenotype of individuals with depression in Alzheimer's.

The present study suffers from its cross-sectional nature and relatively small sample of individuals with a higher level of depressive symptoms. The small number of patients with at least mild depression reflects a sampling bias in the TARC cohort where individuals with high initial levels of depression were excluded from the study. The finding of an upregulation of BDNF related to level of depression needs to be replicated in a larger sample. The present findings are suggestive that individuals with depression in early $\mathrm{AD}$ may represent a subtype that may have a different presentation and may respond differently to pharmacological interventions.

\section{Acknowledgements}

This study was made possible by the Texas Alzheimer's Research Consortium (TARC) funded by the state of
Texas through the Texas Council on Alzheimer's Disease and Related Disorders. Investigators at the University of Texas Southwestern Medical Center at Dallas would also like to acknowledge support from the UTSW Alzheimer's Disease Center NIH, NIA grant P30AG12300.

Declaration of Interest: no authors expressed any conflicts of interest with this project.

\section{REFERENCES}

[1] R. L. Ownby, E. Crocco, A. Acevedo, V. John and P. Loewenstein, "Depression and Risk for Alzheimer Disease: Systematic Review, Meta-Analysis, and Metaregression Analysis," Archives of General Psychiatry, Vol. 63, No. 5, May 2006, pp. 530-538. doi:10.1001/archpsyc.63.5.530

[2] M. Houde, H. Bergman, V. Whitehead and H. Chertkow, "A Predictive Depression Pattern in Mild Cognitive Impairment," International Journal of Geriatric Psychopharmacology, Vol. 23, No.10, October 2008, pp. 1028-1033.

[3] H. B. Lee and C. G. Lyketsos, "Depression in Alzheimer's Disease: Heterogeneity and Related Issues," Biological Psychiatry, Vol. 54, No. 3, August 2003, pp. 353-362. doi:10.1016/S0006-3223(03)00543-2

[4] F. Karege, G. Bondolfi, N. Gervasoni, M. Schwald, J. M. Aubry and G. Bertschy, "Low Brain-Derived Neurotrophic Factor (BDNF) Levels in Serum of Depressed Patients Probably Results from Lowered Platelet BDNF Release Unrelated to Platelet Reactivity," Biological Psychiatry, Vol. 57, No. 9, May 2005, pp. 1068-1072. doi:10.1016/j.biopsych.2005.01.008

[5] C. W. Cotman, "The Role of Neurotrophins in Brain Aging: A Perspective in Honor of Regino Perez-Polo," Neurochemical Research, Vol. 30, No. 6-7, June-July 2005, pp. 877-881. doi:10.1007/s11064-005-6960-y

[6] F. Caraci, A. Copani, F. Nicoletti and F. Drago, "Depression and Alzheimer's Disease: Neurobiological Links and Common Pharmacological Targets," European Journal of Pharmacology, Vol. 626, No. 1, January 2010, pp. 64-71. doi:10.1016/j.ejphar.2009.10.022

[7] A. R. Brunoni, M. Lopes and F. Fregni, "A systematic Review and Meta-Analysis of Clinical Studies on Major Depression and BDNF Levels: Implications for the Role of Neuroplasticity in Depression," International Journal of Neuropsychopharmacology, Vol. 11, No. 8, December 2008, pp. 1169-1180. doi:10.1017/S1461145708009309

[8] F. Karege, G. Perret, G. Bondolfi, M. Schwald, G. Bertschy and J. M. Aubry, "Decreased Serum Brain-Derived Neurotrophic Factor Levels in Major Depressed Patients," Psychiatry Research, Vol. 109, No. 2, March 2002, pp. 143-148. doi:10.1016/S0165-1781(02)00005-7

[9] C. Yasutake, K. Kuroda, T. Yanagawa, T. Okamura and H. Yoneda, "Serum BDNF, TNF-Alpha and IL-1Beta Levels in Dementia Patients: Comparison between Alzheimer's Disease and Vascular Dementia," European Archives of Psychiatry Clinical Neuroscience, Vol. 256, No. 7, Octo- 
ber 2006, pp. 402-406

[10] C. Laske, E. Stransky, T. Letyhe, G. W. Eschweiler, W. Maetzler, A. Wittorf, S. Soekadar, E. Richartz, N. Koehler, M. Bartels, G. Buchkremer and K. Schott, "BDNF Serum and CSF Concentrations in Alzheimer's Disease, Normal Pressure Hydrocephalus and Healthy Controls," Journal of Psychiatric Research, Vol. 41, No. 5, August 2007, pp. 387-394. doi:10.1016/j.jpsychires.2006.01.014

[11] S. E. O’Bryant, B. Hobson, J. R. Hall, S. C. Waring, W. Chan, P. Massman, L. Lacritz, C. Munro Cullum and R. Diaz-Arrastia, "Brain-Derived Neurotrophic Factor Levels in Alzheimer's Disease," Journal of Alzheimers Disease, Vol. 17, No. 2, 2009, pp. 337-341.

[12] C. Laske C, E. Stransky, G. Eschweiler, A. Wittorf, E. Richartz-Salzburger, M. Bartels, G. Buchkremer and K. Schott, "BDNF Serum Concentrations in Patients with Alzheimer's Disease are Associated with Depressive Mood States," Neurology, Psychiatry, and Brain Research, Vol. 12, 2005, pp. 1-4.

[13] J. G. Lee, B. S. Shin, Y. S. You, J. E. Kim, S. W. Yoon, D. W. Jeon, J. H. Baek, S. W. Park and Y. H. Kim, "Decreased Serum Brain-Derived Neurotrophic Factor Levels in Elderly Korean with Dementia," Psychiatry Investigation, Vol. 6, No. 4, December 2009, pp. 299-305. doi:10.4306/pi.2009.6.4.299

[14] S. Waring, S. E. O’Bryant, J. S. Reisch, R. Diaz-Arrastia, J. Knebl and R. Doody, “The Texas Alzheimer's Research Consortium Longitudinal Research Cohort: Study Design and Baseline Characteristics," Texas Public Health Journal, Vol. 60, No. 3, 2008, pp. 9-13.

[15] D. McKhann, D. Drachman, M. Folstein, R. Katzman, C.
Price and E. M. Stadlan, "Clinical Diagnosis of Alzheimer's Disease: Report of the NINCDS-ADRDA Work Group under the Auspices of Department of Health and Human Services Task Force of Alzheimer's Disease," Neurology, Vol. 34, No. 7, July 1984, pp. 939-944.

[16] C. Laske, E. Stransky, T. Leyhe, G. W. Eschweiler, A. Wittorf, E. Richartz, M. Bartels, G. Buchkremer and K. Schott, "Stage-Dependent BDNF Serum Concentrations in Alzheimer's Disease," Journal of Neural Transmission, Vol. 113, No. 9, September 2006, pp. 1217-1224. doi:10.1007/s00702-005-0397-y

[17] F. Angelucci, G. Spalletta, F. di Iulio, A. Ciaramella, F. Salani, L. Colantoni, A. E. Varsi, W. Gianni, G. Sancesario, C. Caltagirone and P. Bossù, “Alzheimer's Disease (AD) and Mild Cognitive Impairment (MCI) Patients are Characterized by Increased BDNF Serum Levels," Current Alzheimer Research, Vol. 7, No. 1, February 2010, pp. 15-20. doi:10.2174/156720510790274473

[18] M. Maes, R. Yirmyia, J. Noraberg, S. Brene, J. Hibbeln, G. Perini, M. Kubera, P. Bob, B. Lerer and M. Maj," The inflammatory \& Neurodegenerative (I\&ND) Hypothesis of Depression: Leads for Future Research and New Drug Developments in Depression," Metabolic Brain Disease, Vol. 24, No. 1, March 2009, pp. 27-53. doi:10.1007/s11011-008-9118-1

[19] S. E. O'Bryant, G. Xiao, R. Barber, J. Reisch, R. Doody, T. Fairchild, P. Adams, S. Waring and R. Diaz-Arrastia, "A Serum Protein-Based Algorithm for the Detection of Alzheimer's Disease," Archives of Neurology, Vol. 67, No. 9, September 2010, pp. 1077-1081. doi:10.1001/archneurol.2010.215 